\title{
On divisibility in definable groups
}

\author{
by
}

Margarita Otero (Madrid)

\begin{abstract}
Let $\mathcal{M}$ be an o-minimal expansion of a real closed field. It is known that a definably connected abelian group is divisible. We show that a definably compact definably connected group is divisible.
\end{abstract}

Let $\mathcal{M}$ be an o-minimal expansion of a real closed field. A group is said to be definable if both the set and the graph of the group operation are definable in $\mathcal{M}$. By results of Pillay in [10], a definable group can be equipped with a definable manifold topology making the group a topological group. Since topological groups are regular spaces, we can suppose that the manifold topology is induced by that of the ambient space (see Theorem 10.1.8 in [6]). In that setting, a definably compact group is a closed and bounded definable group. A definable group is definably connected provided it has no definable subgroups of finite index. A definably connected group which is abelian is also divisible, by Strebonski's Theorem on the finiteness of torsion subgroups (see, e.g., the proof of Theorem 2.1 in [9]).

In this note, using the available literature on both definable groups and topological groups, we prove the following.

THEOREM 1. Let $G$ be a definably compact definably connected definable group. Then $G$ is divisible.

In proving divisibility of the groups we are concerned with, the continuous definable maps $p_{k}: G \rightarrow G: a \mapsto a^{k}$ for $k>0$ will play an important role (in both the Abelian definable case and the classical topological case).

First, we consider the o-minimal cohomology with coefficients in $\mathbb{Q}$, as defined in Section 3 of [9]. Recall that if $X$ is a definable set then $H^{*}(X ; \mathbb{Q})$ is a finite-dimensional $\mathbb{Q}$-vector space such that $H^{m}(X ; \mathbb{Q})=0$ for $m>\operatorname{dim} X$, and moreover $H^{0}(X ; \mathbb{Q}) \cong \mathbb{Q}$ provided $X$ is also definably connected. For an element $x \in H^{m}(X ; \mathbb{Q})$, we say $x$ has degree $m$ and write $\operatorname{deg} x=m$. In [9], 
it is observed that $H^{*}(X ; \mathbb{Q}) \cong \operatorname{Hom}_{\mathbb{Z}}\left(H_{*}(X), \mathbb{Q}\right)$, where $H_{*}(X)$ is the ominimal homology with coefficients in $\mathbb{Z}$. It will be more convenient for our purposes to take o-minimal homology with coefficients in $\mathbb{Q}$. In this case, we also get $H^{*}(X ; \mathbb{Q}) \cong \operatorname{Hom}_{\mathbb{Q}}\left(H_{*}(X ; \mathbb{Q}), \mathbb{Q}\right)$, because $H^{*}(X ; \mathbb{Q}) \cong H_{*}(X) \otimes \mathbb{Q}$ and $\operatorname{Hom}_{\mathbb{Q}}\left(H_{*}(X) \otimes \mathbb{Q}, \mathbb{Q}\right) \cong \operatorname{Hom}_{\mathbb{Z}}\left(H_{*}(X), \mathbb{Q}\right)$.

Notice that $H^{*}(X ; \mathbb{Q})$ is a $\mathbb{Q}$-algebra with product defined as follows: let $d: X \rightarrow X \times X: x \mapsto(x, x)$ be the diagonal map, identify $H^{*}(X \times X ; \mathbb{Q})$ with $H^{*}(X ; \mathbb{Q}) \otimes H^{*}(X ; \mathbb{Q})$ via the o-minimal Künneth formula for cohomology, and let $x \cdot y:=d^{*}(x \otimes y)$ (see [9] for details).

Moreover, we have the following result.

Lemma 2. Let $G$ be a nontrivial definably connected definably compact definable group. Then there is a unique integer $r>0$ and elements $y_{1}, \ldots, y_{r}$ in the o-minimal $\mathbb{Q}$-cohomology algebra of $G$ such that

(i) $\operatorname{deg} y_{i}$ is odd $(i=1, \ldots, r)$,

(ii) $H^{*}(G ; \mathbb{Q})$ is freely generated, as a $\mathbb{Q}$-vector space, by $1\left(\in H^{0}(G ; \mathbb{Q})\right)$ and the monomials $y_{i(1)} \cdot \cdots \cdot y_{i(l)}$ with $1 \leq i(1)<\cdots<i(l) \leq r$.

Proof. By Corollary 3.6 in [9], there is a unique $r \geq 0$ and $y_{1}, \ldots, y_{r}$ satisfying the requirements. Now, since $G$ is definably connected, by Theorem 5.2 in [3], the top o-minimal homology group $H_{n}(G)$ is nontrivial, where $n=\operatorname{dim} G>0$. Therefore, $r>0$.

We write len $x=l$ if $x$ is a monomial of length $l$, i.e., $x=y_{i(1)} \cdot \ldots \cdot y_{i(l)}$ with $1 \leq i(1)<\cdots<i(l) \leq r$ (with the notation of the above lemma). In the following, we are going to consider the maps $p_{k}, k>0$, mentioned above. The computations in [4], for such maps, apply to our o-minimal context and yield the following.

Lemma 3. Let $G$ be a definably connected definable group. For each $k>0$, consider the definable continuous map $p_{k}: G \rightarrow G: a \mapsto a^{k}$ for $a \in G$. Then the map $p_{k}^{*}: H^{*}(G ; \mathbb{Q}) \rightarrow H^{*}(G ; \mathbb{Q})$ sends each monomial $x$ to $k^{\operatorname{len} x} x$.

Proof. See Lemma 5.2 in [9].

Let $X$ be a definable set of dimension $n$ and let $f: X \rightarrow X$ be a continuous definable map. The Lefschetz number of $f$ is defined as follows:

$$
L(f)=\sum_{m=0}^{n}(-1)^{m} \operatorname{trace}\left(f_{*}: H_{m}(X ; \mathbb{Q}) \rightarrow H_{m}(X ; \mathbb{Q})\right) .
$$

(See [5] for the semialgebraic case, and compare with the definition of the Lefschetz number as an intersection number in the o-minimal differentiable case given in [2].) 
Note that the matrix of $f^{*}: H^{m}(X ; \mathbb{Q}) \rightarrow H^{m}(X ; \mathbb{Q})$ is the transpose of the matrix of $f_{*}: H_{m}(X ; \mathbb{Q}) \rightarrow H_{m}(X ; \mathbb{Q})\left(f^{*}=\operatorname{Hom}_{\mathbb{Q}}\left(f_{*}\right)\right)$. Hence, we also get $L(f)=\sum_{m=0}^{n}(-1)^{m} \operatorname{trace}\left(f^{*}: H^{m}(X ; \mathbb{Q}) \rightarrow H^{m}(X ; \mathbb{Q})\right)$.

The next step is to make use of the definable version of the Lefschetz fixed point theorem (see Theorem 1.1 in [8], and Proposition 2 in [5] for the semialgebraic case).

TheOREM 4. Let $X$ be a closed and bounded definably connected set. If $f: X \rightarrow X$ is a continuous definable map and $L(f) \neq 0$, then $f$ has a fixed point.

Corollary 5. Let $G$ be a definably compact definable group. If $f$ : $G \rightarrow G$ is a continuous definable map and $L(f) \neq 0$, then there is an element $b \in G$ such that $f(b)=b$.

Finally, we shall follow Brown [4] to compute $L\left(p_{k}\right)$ for each $k \geq 2$, and prove Theorem 1.

Lemma 6. Let $G$ be a definably connected definably compact definable group. Then, for each $k \geq 2, L\left(p_{k}\right)=(1-k)^{r}$, where $r$ is as in Lemma 2 .

Proof. Let $\left\{1, x_{1}, \ldots, x_{s}\right\}$ be a basis of the $\mathbb{Q}$-vector space $H^{*}(G ; \mathbb{Q})$, where the $x_{i}$ 's are monomials. By Lemma $3, p_{k}^{*}\left(x_{i}\right)=k^{\text {len } x_{i}} x_{i}(i=1, \ldots, s)$ and $p_{k}^{*}(1)=1$. Then the matrix of $p_{k}^{*}: H^{m}(G ; \mathbb{Q}) \rightarrow H^{m}(G ; \mathbb{Q})$ is either 0 (if $H^{m}(G ; \mathbb{Q})=0$ ) or a diagonal matrix with entry $k^{\operatorname{len} x_{i}}$ corresponding to each $x_{i}$ in $H^{m}(G ; \mathbb{Q})$. Therefore, $L\left(p_{k}\right)=\sum_{i=1}^{s}(-1)^{\operatorname{deg} x_{i}} k^{\operatorname{len} x_{i}}+1$. On the other hand, the $x_{i}$ 's are monomials (products of the $y_{j}$ 's of Lemma 2) and the $y_{j}$ 's are of odd degree, so that $\operatorname{deg} x_{i} \equiv \operatorname{len} x_{i}(\bmod 2)$, and hence $L\left(p_{k}\right)=\sum_{i=1}^{s}(-1)^{\operatorname{len} x_{i}} k^{\text {len } x_{i}}+1$. Since there are $\left(\begin{array}{l}r \\ l\end{array}\right)$ monomials of length $l$, we get $L\left(p_{k}\right)=\sum_{l=1}^{r}\left(\begin{array}{l}r \\ l\end{array}\right)(-1)^{l} k^{l}+1=(1-k)^{r}$.

Proof of Theorem 1. Fix $a \in G$ and $k(\geq 2)$. We shall prove the existence of an element $b \in G$ such that $b^{k}=a$. Let $f: G \rightarrow G: c \mapsto c^{k+1} a^{-1}$ for $c \in G$. Since $G$ is definably connected, there is a definable path $\gamma:[0,1] \rightarrow G$ such that $\gamma(0)=a^{-1}$ and $\gamma(1)=e$, where $e$ is the neutral element of $G$. Let

$$
F:[0,1] \times G \rightarrow G:(t, c) \mapsto F(t, c):=c^{k+1} \gamma(t) .
$$

Clearly, $F$ is a definable homotopy between the maps $F(0,-)=f$ and $F(1,-)=p_{k+1}$. Hence, the induced cohomology morphisms $f^{*}$ and $\left(p_{k+1}\right)^{*}$ (both from $H^{*}(G ; \mathbb{Q})$ to $\left.H^{*}(G ; \mathbb{Q})\right)$ ) coincide. Therefore, $L(f)=L\left(p_{k+1}\right)$, and by Lemma $6, L\left(p_{k+1}\right)=(-k)^{r}(\neq 0)$. By Corollary 5 , there is an element $b$ in $G$ such that $b^{k+1} a^{-1}=b$, as required.

After this note was written, two alternative proofs of Theorem 1 have been given in [7] and [1], both papers making reference to a preprint version of this note. 
Acknowledgements. This research was partially supported by GEOR MTM2005-02568 and Grupos UCM 910444.

\section{References}

[1] A. Berarducci, Zero-groups and maximal tori, in: Logic Colloquium 2004, Lecture Notes in Logic 29, Cambridge Univ. Press, 2008, 33-46.

[2] A. Berarducci and M. Otero, Intersection theory for o-minimal manifolds, Ann. Pure Appl. Logic 107 (2001), 87-119.

[3] - , - , Transfer methods for o-minimal topology, J. Symbolic Logic 68 (2003), 785794.

[4] R. Brown, The Lefschetz Fixed Point Theorem, Scott and Foresman, 1971.

[5] G. Brumfiel, A Hopf fixed point theorem for semi-algebraic maps, in: Real Algebraic Geometry (Rennes, 1991), Lecture Notes in Math. 1524, Springer, 1992, 163-169.

[6] L. van den Dries, Tame Topology and o-Minimal Structures, London Math. Soc. Lecture Note Ser. 248, Cambridge Univ. Press, 1998.

[7] M. Edmundo, A remark on divisibility of definable groups, Math. Logic Quart. 51 (2005), 639-641.

[8] - - A fixed point theorem in o-minimal structures, Ann. Inst. Fourier (Grenoble) 57 (2007), 1441-1450.

[9] M. Edmundo and M. Otero, Definably compact abelian groups, J. Math. Logic 4 (2004), 163-180.

[10] A. Pillay, On groups and fields definable in o-minimal structures, J. Pure Appl. Algebra 53 (1988), 239-255.

Departamento de Matemáticas

Universidad Autónoma de Madrid

28049 Madrid, Spain

E-mail: margarita.otero@uam.es

Received 6 October 2008;

in revised form 10 December 2008 